\title{
Lepton Pair Production in High-Frequency Laser Fields ${ }^{1}$
}

\author{
C. Müller*, C. Deneke, M. Ruf, G. R. Mocken, K. Z. Hatsagortsyan, and C. H. Keitel** \\ Max-Planck-Institut für Kernphysik, Saupfercheckweg 1, Heidelberg, 69117 Germany \\ *e-mail: c.mueller@mpi-k.de \\ **e-mail: keitel@mpi-k.de \\ Received November 5, 2008
}

\begin{abstract}
The production of electron-positron and muon-antimuon pairs in high-frequency laser fields via few-photon absorption is considered. It is assumed that an intense X-ray laser beam collides either with a relativistic ion beam or with a second, equally intense laser beam. We study the generation of free $e^{+} e^{-}$pairs, free $\mu^{+} \mu^{-}$pairs, and bound-free $e^{+} e^{-}$pairs where in the latter case the electron is born in a low-lying atomic orbit of the projectile nucleus. Effects resulting from the finite nuclear size, the laser's polarization state, and its magnetic field component are examined, which are testable experimentally by virtue of upcoming X-ray free-electron laser (XFEL) devices.
\end{abstract}

PACS numbers: 12.20.Ds, 21.10.-k, 32.80.Wr, 34.90.+q

DOI: $10.1134 / \mathrm{S} 1054660 \mathrm{X} 09040379$

\section{INTRODUCTION}

High-energy photons can be converted into electron-positron pairs in several ways. In the BreitWheeler process

$$
\gamma_{1}+\gamma_{2} \longrightarrow e^{+} e^{-}
$$

two colliding photons combine their energies to produce the particles $[1,2]$. In the center-of-mass frame the photons have equal energies $\hbar \omega_{\gamma}$ which need to exceed the electron rest mass $m_{e} c^{2}$. The reaction (1) represents the time-reversed annihilation of a free $e^{+} e^{-}$pair into two equal-energy photons. In the Bethe-Heitler process a pair is generated by $\gamma$-photon absorption in the Coulomb field of an atomic nucleus [3, 4]. Here, $\hbar \omega_{\gamma} \geq$ $2 m_{e} c^{2}$ is required. The Bethe-Heitler process is similar to the Breit-Wheeler process (1), but with the second photon being virtual: $\gamma+\gamma^{*} \longrightarrow e^{+} e^{-}$. It was first observed 60 years ago employing energetic Bremsstrahlung photons or nuclear $\gamma$-rays [5]. In the Bethe-Heitler process, due to the presence of the nucleus, the electron can also be created in a bound atomic state [6-8]. Then the required photon energy is reduced by the binding energy. At higher photon energies also heavier particles such as muon-antimuon $\mu^{+} \mu^{-}$ pairs can be produced [9].

The Breit-Wheeler and Bethe-Heitler mechanisms of pair production possess nonlinear generalizations when the photon source is an intense laser beam (see the recent reviews $[10,11])$. Then multiphoton processes are possible where more than one photon of the same sort (i.e., with the same four-momentum and polarization) participate in the pair production. The nonlinear Breit-Wheeler process

\footnotetext{
${ }^{1}$ The article is published in the original.
}

$$
\gamma+n \omega_{L} \longrightarrow e^{+} e^{-}
$$

was observed in the late 1990s at SLAC (Stanford, USA) with a high-energy Compton photon of $\hbar \omega_{\gamma} \approx$ $30 \mathrm{GeV}$ and $n=5$ optical laser photons of $\hbar \omega_{L} \approx 2 \mathrm{eV}$ $[12,13]$. In the center-of-mass frame this implies $\hbar \omega_{\gamma}^{\prime}=5 \hbar \omega_{L}^{\prime} \approx m_{e} c^{2}$. Nonlinear Bethe-Heitler pair creation has been studied by several theoreticians (see [14-19] and references therein), with a focus on the tunneling regime of the process, but has not been observed in experiment yet. The process - in particular its bound-free channel-is formally related to multiphoton ionization of atoms which has been investigated intensively in experiment and theory [20, 21]. Bound-free Bethe-Heitler pair creation can be viewed as the recombination (i.e., time-reversed ionization) of an electron from the negative-energy Dirac continuum into a bound atomic state.

In this paper we discuss various nonlinear schemes of lepton pair production in intense high-frequency laser fields. The multiphoton regime is considered where the laser intensity parameter is small: $\xi=$ $e E_{L} / m_{e} c \omega_{L} \lesssim 1$, with the electron charge $e$, the laser peak field strength $E_{L}$, and the speed of light $c$. Largescale XFEL facilities currently under construction at SLAC and DESY (Hamburg, Germany) are envisaged to obtain photon energies of $\hbar \omega_{L} \sim 10 \mathrm{keV}$ at intensities up to $I_{L} \sim 10^{20} \mathrm{~W} / \mathrm{cm}^{2}\left(\xi \sim 10^{-3}\right)$ [22]. In Section 2 we consider few-photon Bethe-Heitler pair creation of muons in collisions of ultrarelativistic bare ions with XFEL radiation: $\gamma^{*}+n \omega_{L} \longrightarrow \mu^{+} \mu^{-}$. The high Lorentz factor of the ions is exploited to boost the laser photon energy to larger values in the ionic rest frame. The nuclear size is shown to have substantial impact on the production rates. In Section 3 the generation of bound 
electrons and free positrons in relativistic ion-XFEL collisions is treated. Here the influences of excited atomic states and of the laser polarization are addressed. Finally, we consider in Section 4 the creation of $e^{+} e^{-}$pairs in counterpropagating laser beams of equal intensity and frequency. In this situation the pair is created by "doubly nonlinear" variants

$$
n_{1} \omega_{1}+n_{2} \omega_{2} \longrightarrow e^{+} e^{-}
$$

of the Breit-Wheeler reaction (1), where $n_{1}$ photons are absorbed from the first beam and $n_{2}$ photons from the second. We finish with a conclusion in Section 5.

It is noteworthy that in laser-ion and laser-laser collisions also other nonlinear quantum electrodynamical processes than pair creation can occur. For example, recent theoretical studies have considered photon-splitting [23], photon-fusion [24], harmonic generation [25], and Delbrück scattering [26]. They usually assume superintense laser fields of low frequency where the quantum vacuum is polarized by the quasistatic electric field of the laser. For recent pair creation studies in low-frequency laser fields by the Schwinger tunneling mechanism we refer to [27] where also further references can be found.

\section{MUON PAIR CREATION IN LASER-ION COLLISIONS}

In this section we consider the nonlinear creation of a free $\mu^{+} \mu^{-}$pair in the collision of an X-ray laser beam $\left(\hbar \omega_{L}=12 \mathrm{keV}\right)$ with an ultrarelativistic ion beam $\left(\gamma_{p}=\right.$ 7000) [28]. This parameter combination would be achieved in a hypothetical combination of the upcoming XFEL sources with the nuclear beam from the recently commissioned LHC at CERN (Geneva, Switzerland). In the ion frame the photon energy amounts to $\hbar \omega_{L}^{\prime} \approx 2 \gamma_{p} \hbar \omega_{L}=168 \mathrm{MeV}$. The energy gap of $2 m_{\mu} c^{2}$ for muon pair production can thus be overcome by twophoton absorption; $m_{\mu} \approx 207 m_{e}$ denotes the muon mass. The large muon mass is connected with a correspondingly small Compton wavelength $\chi_{c}^{(\mu)} \approx 1.86 \mathrm{fm}$, which is smaller than the radius of most nuclei. Therefore the finite nuclear size must be taken into account.

The standard theoretical approach to the creation of free lepton pairs in combined laser and Coulomb fields $[17,18]$ employs the strong-field approximation (SFA) [20]. In the nuclear rest frame the corresponding transition amplitude reads

$$
S_{\mathrm{ff}}^{\mathrm{SFA}}=-\frac{i e}{\hbar c} \int d^{4} x \bar{\Psi}_{p_{-}}^{(\mathrm{V})} \gamma_{0} A_{\mathrm{nuc}}^{0}(r) \Psi_{p_{+}}^{(\mathrm{V})} .
$$

The muons are described by relativistic Volkov states $\Psi_{p_{ \pm}}^{(\mathrm{V})}$ with free momenta $\mathbf{p}_{ \pm}$[29]. The use of Volkov states allows to include the interaction of the leptons with the plane-wave laser field up to all orders. The nuclear field $A_{\text {nuc }}^{0}(r)$ is taken into account within the first-order of perturbation theory. $\gamma_{0}$ denotes a Dirac $\gamma$ matrix. In this paper, the nucleus is assumed to be a homogenously charged sphere. This simple model provides a rather good approximation to real nuclear charge densities, in particular for heavy isotopes. An alternative parametrization is represented by a Gaussian distribution which has been applied in [28]; the specific choice of the nuclear model has only minor influence on the results. The radius $a$ of the sphere is related to the nuclear rms. charge radius by $r_{\mathrm{rms}}=\sqrt{3 / 5} a$. This leads in Eq. (4) to the appearance of the corresponding elastic nuclear form factor

$$
F\left(q^{2}\right)=\frac{3}{\alpha^{3}}(\sin \alpha-\alpha \cos \alpha),
$$

with $\alpha=q a / \hbar$ and the momentum transfer to the nucleus $\mathbf{q}=\tilde{\mathbf{p}}_{+}+\tilde{\mathbf{p}}_{-}-2 \hbar \mathbf{k}$. In the multiphoton regime $\left(\xi_{\mu}=\xi_{m_{e}} / m_{\mu} \ll 1\right)$, the quasi-momenta $\tilde{\mathbf{p}}_{ \pm}$of the muons practically coincide with their free momenta $\mathbf{p}_{ \pm}$[29], and $\mathbf{k}$ is the laser wave vector. The form factor (5) exhibits an oscillatory nature which is characteristic for most heavy nuclei [30]. The form factor $F\left(q^{2}\right)$ leads to substantial reduction of the production rate when the recoil $q \sim m_{\mu} c$ exceeds $\hbar / a$ (i.e., when $\alpha \gg 1$ ).

The fully differential elastic rate for two-photon muon pair creation is obtained by summing the square of the amplitude (4) over the muon spin states:

$d R_{\mathrm{el}}=\sum_{\text {spins }}\left|S_{\mathrm{ff}}^{\mathrm{SFA}}\right|^{2} \frac{d^{3} p_{+}}{(2 \pi \hbar)^{3}} \frac{d^{3} p_{-}}{(2 \pi \hbar)^{3}}=d R_{0} Z^{2} F^{2}\left(q^{2}\right),(6)$

where $d R_{0}$ denotes the differential production rate for a pointlike proton and $Z$ is the nuclear charge number. Equation (6) is called the elastic production rate since the nucleus is assumed to remain in its ground state. An additional contribution $d R_{\text {inel }} \approx d R_{0} Z\left[1-F^{2}\left(q^{2}\right)\right]$ to the process stems from the inelastic channel $[9,31]$, where the recoil leads to nuclear excitation. The total rate is $R_{\mathrm{tot}}=R_{\mathrm{el}}+R_{\text {inel }}$. Below we show angular muon spectra as well as elastic and total production rates; all results refer to the rest frame of the projectile.

Figure 1 demonstrates the influence of the nuclear extension by showing the angular distribution of the muons in the elastic production channel. Various nuclei are considered; the respective rms charge radii amount to $0.875 \mathrm{fm}$ (proton), $2.470 \mathrm{fm}\left({ }^{12} \mathrm{C}\right), 4.188 \mathrm{fm}\left({ }^{84} \mathrm{Kr}\right)$, and $5.851 \mathrm{fm}\left({ }^{238} \mathrm{U}\right)$ [32]. The result for a pointlike nucleus is also shown for comparison. The rates are strongly reduced with increasing nuclear size because the muons are created typically at distances $r \sim \chi_{c}^{(\mu)}$ inside the nucleus where they experience a reduced nuclear field. Moreover, for large nuclei the oscillating character of the form factor becomes apparent in the 


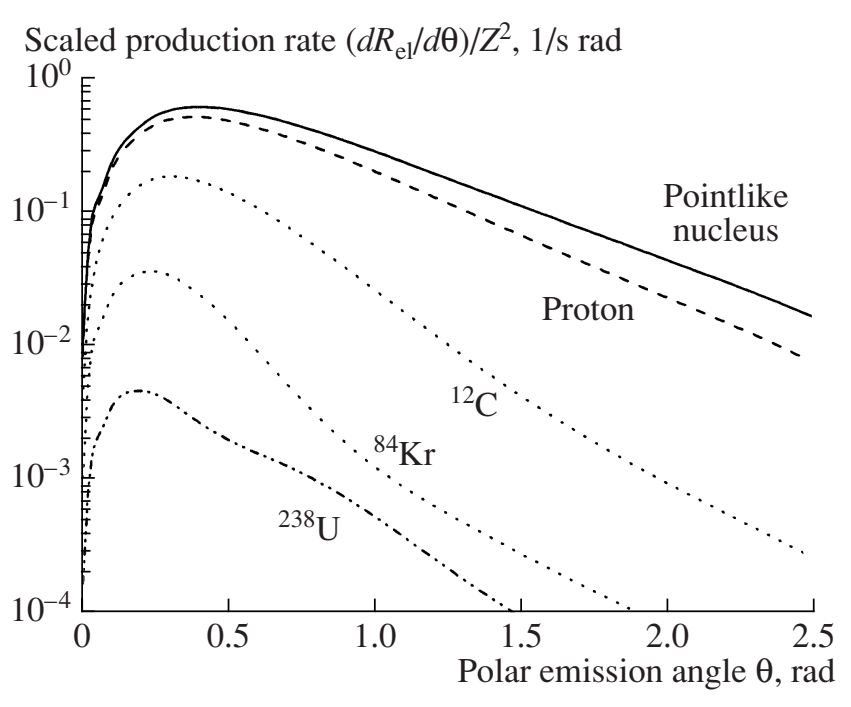

Fig. 1. Angular spectra of one of the muons produced by two-photon absorption from an intense XFEL beam $\left(\hbar \omega_{L}=\right.$ $12 \mathrm{keV}, I_{L}=2.5 \times 10^{22} \mathrm{~W} / \mathrm{cm}^{2}$ ) colliding with various ultrarelativistic nuclei $\left(\gamma_{p}=7000\right)$. The angle is measured with respect to the laser beam axis. Note that the rates are scaled by the nuclear charge.

spectra. Pronounced nuclear size effects are also known from muon production by single $\gamma$-photon impact on nuclei [9] and in relativistic heavy-ion collisions [33].

Figure 2 shows integrated rates of $\mu^{+} \mu^{-}$production for several projectiles. For a pointlike proton, the total rate coincides with the elastic rate and amounts to $R_{0} \approx$ $0.58 \mathrm{~s}^{-1}$ (cp. [16, Eq. (26)]). For an extended projectile the elastic rate increases with its charge as $Z^{2}$ but decreases with its size. This interplay leads to the emergence of maximum elastic rates for medium-heavy ions $(Z \approx 60)$. The same maximum position has been found in [28]. We note that a maximum also arises for elastic muon production by a single photon of twice the energy. The total rate $R_{\text {tot }}$ does not exhibit a maximum but saturates at high $Z$ values since the inelastic contribution increases with nuclear charge. The total rates are still considerably smaller than the point-nucleus results. For heavy ions the main contribution stems from the inelastic channel where the protons inside the nucleus act incoherently $\left(R_{\text {inel }} \propto Z\right)$. This implies that despite the high charges, Coulomb corrections to the SFA treatment are of minor importance [33, 34]. In the laboratory frame, the production rates of Fig. 2 are reduced by a factor $\gamma_{p}^{-1}$ due to relativistic time dilation and, accordingly, reach values of about $R_{\mathrm{lab}} \approx 10^{-2} \mathrm{~s}^{-1}$.

\section{BOUND-FREE $e^{+} e^{-}$PAIR CREATION IN LASER-ION COLLISIONS}

Electron-positron pairs can be generated in XFELion collisions already at lower projectile energies $\left(\gamma_{p} \geqslant\right.$ 50). Here, the nuclear size does not play a role since the

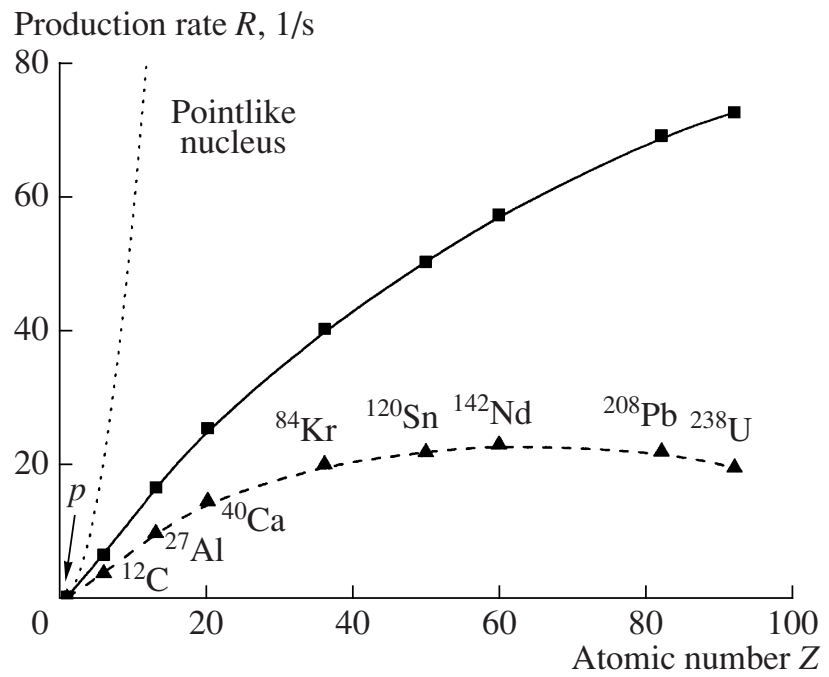

Fig. 2. Integrated rates for muon pair creation in XFEL-ion collisions as in Fig. 1. The triangles show elastic rates, whereas the squares indicate total ("elastic + inelastic") rates. The numerical data are connected by fit curves. The dotted line holds for a pointlike nucleus.

electron Compton wavelength $\sim \chi_{c}^{(e)}$ is larger by two orders of magnitude than the nuclear radius. In the following we consider the bound-free channel of the nonlinear Bethe-Heitler process [35-37], with the electron born in the atomic K-shell or L-shell. Within the SFA, the transition amplitude can be written in the post-form as

$$
S_{\mathrm{bf}}^{\mathrm{SFA}}=-\frac{i e}{\hbar c} \int d^{4} x \bar{\phi}\left(\boldsymbol{\gamma} \cdot \mathbf{A}_{L}\right) \Psi_{p_{+}}^{(\mathrm{V})} .
$$

Here, $\phi$ is the bound Coulomb-Dirac state of the electron, $\Psi_{p_{+}}^{(\mathrm{V})}$ is the Dirac-Volkov state of the positron, and $\mathbf{A}_{L}$ denotes the laser vector potential. We note that Eq. (7) is similar to the SFA amplitude for strong-field ionization in the prior-form [20]. Due to the presence of a bound state in Eq. (7), the evaluation of the pair production rate can be carried out by analytical means to a large extent. The calculation was first performed for circular laser polarization [35], and recently generalized to a linearly polarized beam [36]. We show below results for the angular distributions of the emitted positron and for the total production rate in the rest frame of the nucleus. The reaction proceeds nonlinearly by the absorption of two or more laser photons.

Figure 3 shows the distribution of the created positrons with respect to the polar angle between the positron momentum and the laser propagation direction. The electron is captured to the K-shell. The positrons are emitted predominantly under small angles around $\vartheta \approx 15^{\circ}$ in the ion frame. In this angular range the spectrum is slightly enhanced for linear as compared to circular laser polarization at a given intensity. 


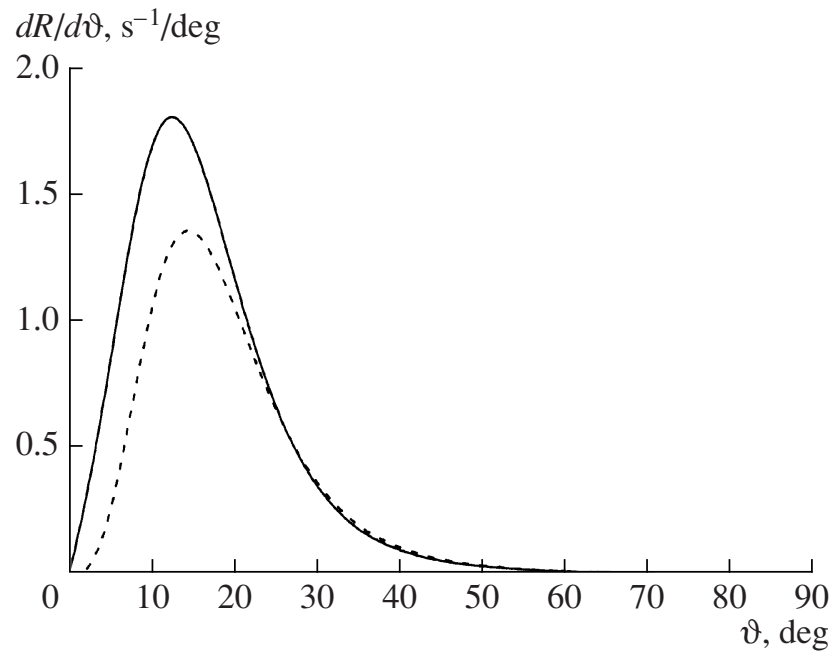

Fig. 3. Comparison of the polar angular spectra for linear (solid line) versus circular (dashed line) laser polarization. $\vartheta$ denotes the angle between the positron momentum and the laser photon momentum. The collision parameters are $\hbar \omega_{L}=9 \mathrm{keV}, I_{L}=7.2 \times 10^{17} \mathrm{~W} / \mathrm{cm}^{2}, Z=50, \gamma_{p}=50, n=2$.

The electron is created in the $1 s$ ground state.

At larger angles both polarization states yield identical spectra. The total integrated rate amounts to $R_{1 s}=$ $34.4 \mathrm{~s}^{-1}$ for linear polarization; for circular polarization it is slightly smaller. The reason for the difference lies in the higher peak electric field strength of the linearly polarized beam and the nonlinear character of the pro-

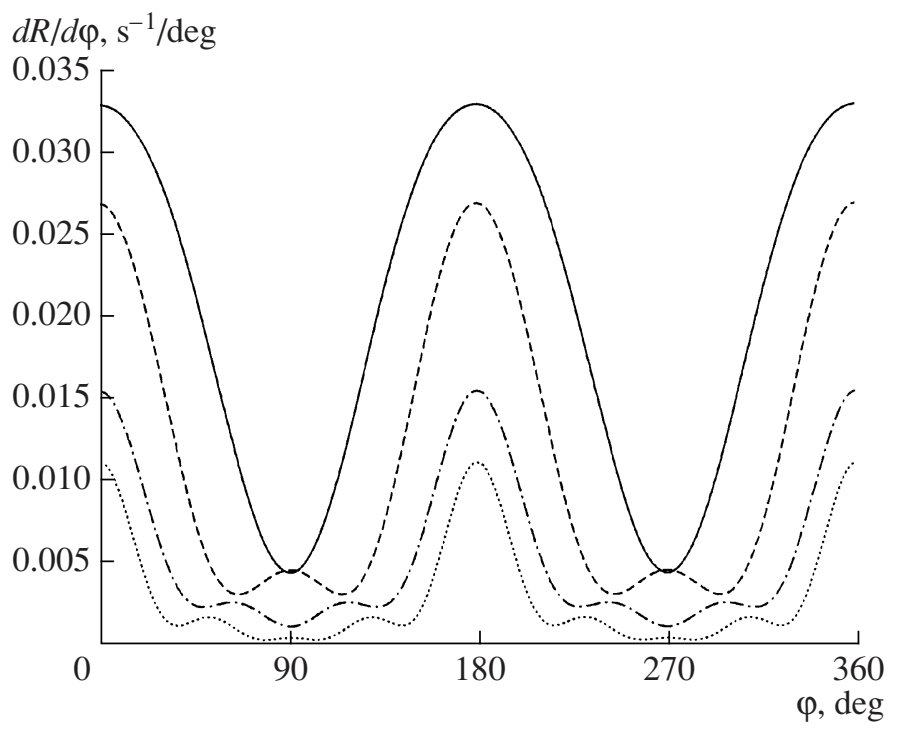

Fig. 4. Azimuthal angular spectra for pair production with electron capture to the $2 s$ state. Shown is the distribution for absorption of two (solid line), three (dashed line), four (dashed-dotted line), and five (dotted line) photons from the linearly polarized wave. The other collision parameters are as in Fig. 3. The values are scaled in order to accommodate all curves in one plot. cess. For pair creation by a single photon the total rate is independent of the photon polarization. In the laboratory frame the total rates are reduced by a time dilation factor $\gamma_{p}^{-1}$.

While for a circularly polarized laser beam the azimuthal distribution of the positrons is isotropic, structure arises for a linearly polarized field (see Fig. 4). The emission is maximal along the laser electric field component and minimal in the magnetic field direction. For higher photon orders with $n \geq 3$ additional structures appear. In particular, the position of the minimum is shifted for odd photon numbers. We note that the higher photon orders are suppressed by a factor of $\xi^{2 n}$ (here $\left.\xi=10^{-4}\right)$.

The contribution to the total production rate from the L-shell is rather small, since the $\mathrm{K}$-shell has a larger width in momentum space. The relative contributions for various nuclear charge numbers are shown in Fig. 5. Capture processes occur preferentially into $s$-states, which obey a $Z^{5}$ scaling law. For the $2 s: 1 s$ ratio we find an approximately constant value of $1 / 8$. This is in agreement with a general rule known, for example, from one-photon pair production in the high-energy limit [7], according to which the contribution from the $s$-state with principal quantum number $n_{p}$ is reduced by the factor $n_{p}^{-3}$ as compared to the $1 s$ contribution. For $Z=50$ the rate amounts to $R_{2 s}=4.33 \mathrm{~s}^{-1}$. The $2 p_{1 / 2}$ state contributes by three orders of magnitude less than the $2 s$ state at low projectile charges. At high $Z$ values,

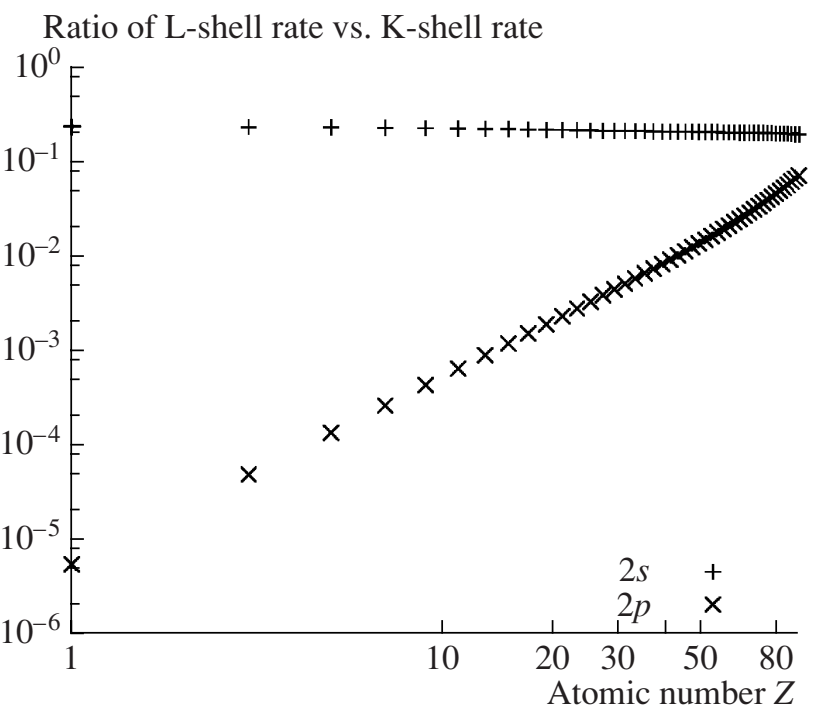

Fig. 5. Contributions of the $2 s$ and $2 p_{1 / 2}$ states to the total pair production rate as a function of the projectile charge. The values are given as fractions of the dominant K-shell contribution. The remaining collision parameters are as in Fig. 3, with a linearly polarized XFEL beam. 
however, its contribution becomes appreciable as the scaling with $Z^{7}$ is steeper here. The reason is that the lower components of the bound Coulomb-Dirac state exhibit $s$-wave character and become comparable in size with the upper components for high nuclear charges.

Concluding this section we compare the SFA results for bound-free pair creation by a single photon in the high-energy limit $\left(\omega_{L} \longrightarrow \infty\right)$ with corresponding calculations by Pratt and Gavrila [7] (obtained in photoionization studies) and by Agger and Sørensen [8]. Those include Coulomb corrections on the outgoing positron wave function by employing distorted waves (i.e., plane waves modified by a Coulomb phase) or Coulomb-Dirac continuum states, respectively. Figure 6 shows that the SFA approach agrees well with the other calculations at low projectile charges but slightly overestimates the $2 s: 1 s$ ratio by about $10 \%$ at high $Z$ values, where Coulomb effects are appreciable.

\section{4. $e^{+} e^{-}$PAIR CREATION \\ IN COUNTERPROPAGATING LASER BEAMS}

Finally, we briefly discuss the generation of $e^{+} e^{-}$ pairs in two counterpropagating laser pulses of equal frequency and intensity. The two laser pulses form a standing wave which, in the high-field region, can be approximated by a purely electric field oscillating in time (dipole approximation). This approach is expected to be justified when the spatial scale of the field variation $\sim \lambda_{L}=2 \pi c / \omega_{L}$ is much larger than both the pair formation length $\sim \chi_{c}^{(e)}$ and the coherence length $l_{\text {coh }}=$ $m c^{2} / e E$. Many theoreticians have calculated the process this way (for a comprehensive list of references see [10, Section $7.1 .3 ; 27])$. The production process exhibits a resonant nature when the total energy of the created pair is an integral multiple of the laser frequency. Then a characteristic Rabi flopping between the negativeenergy and the positive-energy Dirac continua is taking place [38]. This dynamics is analogous to the oscillating population of an atomic two-level system coupled resonantly to an external field.

By employing the same approach based on the dipole approximation we have investigated the nonperturbative multiphoton regime of the process where $\xi$ 1 [39]. We found that the relative width $\Delta \omega$ of the resonance peaks follows a power law: $\Delta \omega \sim n^{-\beta}$, with the multiphoton order of the process $n=n_{1}+n_{2}$ [cf. (3)] and $\beta \approx 5$. This kind of behaviour is known from the general theory of parametric resonances. Moreover, the pair production rate is exponentially suppressed by a factor $\sim \exp \left(-3 m_{e} c^{2} / \hbar \omega_{L}\right)$ in optical laser fields, but significantly enhanced in radiation fields of high frequency. In fact, a promising opportunity for an experimental observation of the process is provided by the upcoming XFEL sources [40]. This prospect calls for calculations involving the spatial field dependence and, thus, the

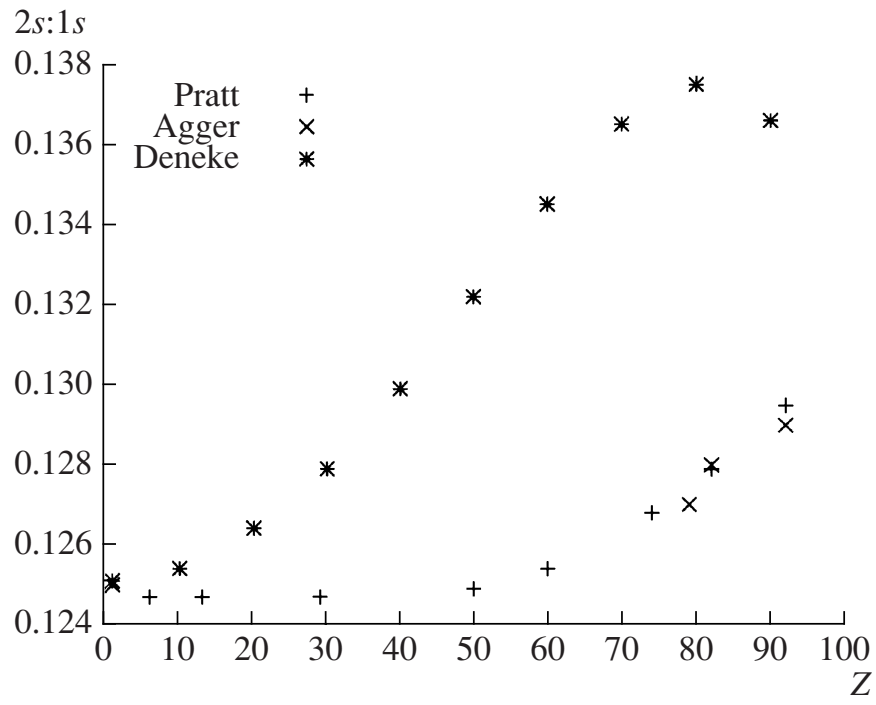

Fig. 6. Ratio of the contributions from the $2 s$ and $1 s$ states to bound-free pair creation by a single photon of infinite energy. Shown is a comparison of the $Z$-dependence resulting from three different calculations (see text for details).

laser magnetic field. Calculation of quantum processes in external fields with separate space and time dependences is in general a very challenging task [41]. In the present case it has been accomplished by numerical means utilizing an advanced computer code [42] for solving the corresponding Dirac equation. This allows us to study the influence of the laser magnetic field on the Rabi flopping dynamics and the total pair production probability [43].

\section{CONCLUSIONS}

The generation of $e^{+} e^{-}$and $\mu^{+} \mu^{-}$pairs by few-photon absorption from intense laser waves of high frequencies has been surveyed. The generation of muon pairs in ultrarelativistic ion-XFEL collisions proceeds via the nonlinear Bethe-Heitler mechanism and is strongly suppressed by the finite nuclear size. For bare uranium the rate is reduced by two orders of magnitude as compared to a point nucleus. In moderately relativistic ionXFEL collisions bound-free $e^{+} e^{-}$pair production can occur by the absorption of two photons. We have shown that electron capture to the K-shell gives the main contribution $(\approx 85 \%)$ to this process. Besides, linear laser polarization leads to slightly larger production rates than circular polarization and to rich structures in the azimuthal positron distribution at high photon orders. In the Breit-Wheeler-type process of $e^{+} e^{-}$pair creation in counterpropagating laser beams the production probability depends on the pulse duration in an oscillatory manner. At high laser frequencies the impact of the laser magnetic field on this Rabi population dynamics is of interest. 


\section{REFERENCES}

1. G. Breit and J. A. Wheeler, Phys. Rev. 46, 1087 (1934).

2. J. M. Jauch and F. Rohrlich, The Theory of Photons and Electrons (Springer, New York, 1976).

3. H. A. Bethe and W. Heitler, Proc. R. Soc. London A 146, 83 (1934); H. A. Bethe and L. C. Maximon, Phys. Rev. 93, 768 (1954); W. Heitler, The Quantum Theory of Radiation (Clarendon, Oxford, 1954).

4. J. W. Motz, H. A. Olsen, and H. W. Koch, Rev. Mod. Phys. 41, 581 (1969).

5. G. P. Adams, Phys. Rev. 74, 1707 (1948); R. L. Walker, Phys. Rev. 76, 527 (1949); J. L. Lawson, Phys. Rev. 75, 433 (1949).

6. The results on photoionization by F. Sauter, Ann. Physik 11, 454 (1931) can be transformed into bound-free pair creation rates via the usual crossing symmetry.

7. R. H. Pratt, Phys. Rev. 119, 1619 (1960); M. Gavrila, Phys. Rev. 124, 1132 (1961).

8. C. K. Agger and A. H. Sørensen, Phys. Rev. A 55, 402 (1997).

9. A. Alberigi-Quaranta et al., Phys. Rev. Lett. 9, 226 (1962); Y.-S. Tsai, Rev. Mod. Phys. 46, 815 (1974).

10. Y. I. Salamin, S. X. Hu, K. Z. Hatsagortsyan, and C. H. Keitel, Phys. Rep. 427, 41 (2006).

11. M. Marklund and P. K. Shukla, Rev. Mod. Phys. 78, 591 (2006); G. A. Mourou, T. Tajima, and S. V. Bulanov, Rev. Mod. Phys. 78, 309 (2006).

12. D. Burke, R. C. Field, G. Horton-Smith, et al., Phys. Rev. Lett. 79, 1626 (1997).

13. H. R. Reiss, J. Math. Phys. 3, 59 (1962); Phys. Rev. Lett. 26, 1072 (1971); V. I. Ritus, Nucl. Phys. B 44, 236 (1972); J. Sov. Laser Res. 6, 497 (1985); http:// www.springerlink.com/content/r28rq38u6587v572.

14. V. P. Yakovlev, Zh. Eksp. Teor. Fiz. 49, 318 (1965) [Sov. Phys. JETP 22, 223 (1966)].

15. S. P. Roshchupkin, Laser Phys. 6, 837 (1996).

16. A. I. Milstein, C. Müller, K. Z. Hatsagortsyan, et al., Phys. Rev. A 73, 062106 (2006).

17. J. Z. Kaminski, K. Krajewska, and F. Ehlotzky, Phys. Rev. A 74, 033402 (2006); K. Krajewska and J. Z. Kaminski, Laser Phys. 18, 185 (2008).

18. M. Yu. Kuchiev and D. J. Robinson, Phys. Rev. A 76, 012107 (2007).

19. E. Lötstedt, U. D. Jentschura, and C. H. Keitel, Phys. Rev. Lett. 101, 203001 (2008).

20. H. R. Reiss, Prog. Quant. Electron. 16, 1 (1992).

21. W. Becker, F. Grasbon, R. Kopold, et al., Adv. At., Mol., Opt. Phys. 48, 35 (2002); D. B. Milošević and F. Ehlotzky, Adv. At. Mol. Opt. Phys. 49, 373 (2003).

22. L. F. DiMauro, J. Arthur, N. Berrah, et al., J. Phys. Conf. Ser. 88, 012058 (2007); M. Altarelli, R. Brinkmann, M. Chergui, et al., Technical Design Report of the European XFEL, DESY 2006-097; http://www.xfel.net.

23. A. Di Piazza, A. I. Milstein, and C. H. Keitel, Phys. Rev. A 76, 032103 (2007); G. Brodin, M. Marklund, B. Eliasson, and P. K. Shukla, Phys. Rev. Lett. 98, 125001 (2007).

24. A. Di Piazza, K. Z. Hatsagortsyan, and C. H. Keitel, Phys. Rev. Lett. 100, 010403 (2008).

25. A. Di Piazza, K. Z. Hatsagortsyan, and C. H. Keitel, Phys. Rev. D 72, 085005 (2005); A. Fedotov and N. Narozhny, Phys. Lett. A 362, 1 (2007).
26. A. Di Piazza and A. I. Milstein, Phys. Rev. A 77, 042102 (2008).

27. S. S. Bulanov, N. B. Narozhny, V. D. Mur, and V. S. Popov, Zh. Eksp. Teor. Fiz. 129, 14 (2006) [JETP 102, 9 (2006)]; M. V. Fedorov, M. A. Efremov, and P. A. Volkov, Opt. Comm. 264, 413 (2006); D. B. Blaschke, A. V. Prozorkevich, C. D. Roberts, et al., Phys. Rev. Lett. 96, 140402 (2006); M. Y. Kuchiev, Phys. Rev. Lett. 99, 130404 (2007); N. I. Chott, Q. Su, and R. Grobe, Phys. Rev. A 76, 010101 (2007); T. Cheng, N. I. Chott, Q. Su, and R. Grobe, Laser Phys. 18, 190 (2008); F. Hebenstreit, R. Alkofer, and H. Gies, Phys. Rev. D 78, 061701 (2008); R. Schützhold, H. Gies, and G. Dunne, Phys. Rev. Lett. 101, 130404 (2008).

28. C. Müller, C. Deneke, and C. H. Keitel, Phys. Rev. Lett. 101, 060402 (2008).

29. V. B. Berestetskii, E. M. Lifshitz, and L. P. Pitaevskii, Relativistic Quantum Theory (Pergamon, Oxford, 1971).

30. B. Povh, K. Rith, and C. Scholz, Particles and Nuclei (Springer, Berlin, 2006).

31. L. D. Landau and E. M. Lifshitz, Quantum Mechanics (Pergamon, Oxford, 1965).

32. I. Angeli, At. Data Nucl. Data Tables 87, 185 (2004); P. J. Mohr and B. N. Taylor, Rev. Mod. Phys. 77, 1 (2005).

33. J. Eichler, Phys. Rep. 193, 165 (1990); G. Baur, K. Hencken, and D. Trautmann, Phys. Rep. 453, 1 (2007).

34. A. B. Voitkiv, private communication.

35. C. Müller, A. B. Voitkiv, and N. Grün, Phys. Rev. Lett. 91, 223601 (2003).

36. C. Deneke and C. Müller, Phys. Rev. A 78, 033431 (2008).

37. V. I. Matveev, E. S. Gusarevich, and I. N. Pashev, Zh. Eksp. Teor. Fiz. 127, 1187 (2005) [JETP 100, 1043 (2005)].

38. V. S. Popov, Pis'ma Zh. Eksp. Teor. Fiz. 18, 435 (1973) [JETP Lett. 18, 255 (1973)]; N. B. Narozhny and A. I. Nikishov, Zh. Eksp. Teor. Fiz. 65, 862 (1973) [Sov. Phys. JETP 38, 427 (1974)]; V. M. Mostepanenko and V. M. Frolov, Yad. Fiz. 19, 885 (1974) [Sov. J. Nucl. Phys. 19, 451 (1974)]; H. K. Avetissian, A. K. Avetissian, G. F. Mkrtchian, and Kh. V. Sedrakian, Phys. Rev. E 66, 016502 (2002).

39. G. R. Mocken, M. Ruf, C. Müller, and C. H. Keitel, in preparation.

40. A. Ringwald, Phys. Lett. B 510, 107 (2001).

41. H. Gies and K. Klingmüller, Phys. Rev. D 72, 065001 (2005); G. V. Dunne and C. Schubert, Phys. Rev. D 72, 105004 (2005).

42. G. R. Mocken and C. H. Keitel, Comput. Phys. Comm. 178, 868 (2008).

43. M. Ruf, G. R. Mocken, C. Müller, K. Z. Hatsagortsyan, and C. H. Keitel, submitted. 